\title{
Industrial Automation using loT with Raspberry Pi
}

\author{
H. K. Merchant \\ PG Student, \\ Department of Electronics and \\ Telecommunication, \\ Matoshri college Engineering and Research \\ center, Nashik Savitrabai Phule Pune University, \\ Pune, India
}

\author{
D. D. Ahire \\ Assistant Professor, \\ Department of Electronics and \\ Telecommunication, \\ Matoshri college Engineering and Research \\ center, Nashik Savitrabai Phule Pune University, \\ Pune, India
}

\begin{abstract}
Internet of Things (IoT) is propagating and blooming technology, in previous years. IoT is the collection of the sensors data through embedded system and this embedded system upload the data on internet. There are many challenges to IoT and Industrial Automation for example Data and service security, Trust, data integrity, information privacy, scalability and interoperability Automation Domain Constrains. This paper combines the concept of Raspberry Pi Industrial workstation and Industrial Automation using IoT. The system uses the raspberry pi as controller and server, the programing is done in the python language. The webpage is designed in HTML, JQuery, ajax and Flask as framework for rendering the HTML template in python. All sensor data are collected through raspberry pi. All the use full data are access remotely through internet of thing platform. Here the blade ageing system of cutter tool is taken to as industrial example and current is monitored to the webpage using raspberry pi as server. This system demonstrates successful measurement of current consumption of cutting tool and indicate to change the blade if it damaged. It also senses the workstation temperature.
\end{abstract}

\section{Keywords}

IoT, Industrial Internet of Things(IIOT), Wireless Sensor Network, Raspberry Pi.

\section{INTRODUCTION}

A large range of industrial IoT application are developed within last few years. It was initiated from RFID technology, where microchips transmit the identification information to a reader through wireless communication. And further technology goes to the wireless sensor networks (WSNs), which mainly use interconnected intelligent sensors to sense and for monitoring. Internet of Things (IoT) is a concept that considers pervasive presence in the environment of a variety of things/objects that through wireless and wired connections and unique addressing schemes are able to interact with each other and cooperate with other things/objects to create new applications, services and reach common goals. The IoT applications are; smart cities, smart energy and the smart grids, smart transportation and enabling traffic management and control.

The $\mathrm{R}-\mathrm{Pi}$, is a single-board computer which is uses Linux based OS and that can be directly used in electronics projects because it has general purpose input/output (GPIO) pins right on the board

This project involves the detail design and construction of an Industrial automation system using Raspberry Pi board and Internet connection. The automation may be semi or fully controlled and monitors the utility grid connected sensors. This project is a demonstration of how to design and build a multipurpose remotely controlled system that can switch any industrial acuter by accessing an Raspberry pi, which is programmed to control the systems inside industrial environment, when the person is away from work station and enable a person to get the related information on phone. The system will provide feedback indicating the current state of the system stopped or functioning.

\subsection{Problem Formulation}

The goal of the system is to create a small industrial environment, where sensor can be monitor and controlled only based on the data received from the industrial site sensors. This data is processed and control action placed accordingly.

The system has to be implemented in a loop, so no human intervention is allowed. This means that the system is adaptive to the environment.

The whole control device of the system it's a small Raspberry PI. This computing system its used due to the fact to prove that the whole industrial system can be automated just with a card size computing system or a microchip and the industrial environment data can be viewed or monitored from IoT.

\subsection{Objective}

1. To make Smart Industrial environment which enables the user to monitor and control industria parameter on real time basis using Mobile device.

2. To save the energy of the workstation by getting the conclusion of the cutter tools blade ageing system through web page.

The flow of the paper is as follows: Section II describes the related work in the industrial automation. Merits \& Challenges of the systems are described in section III and in section IV the proposed system is explained. In section V result is shown, in section VI overall paper is concluded.

\section{RELATED WORK}

In this section, the related work of IIOT is explained. There are many ways to developed the system. In Survey paper [1], system is based on raspberry pi which enables the costeffective environment monitoring. The author uses the python programming language to control the industrial parameters and access them through IoT and Xively is the IoT platform which is used in this paper. 


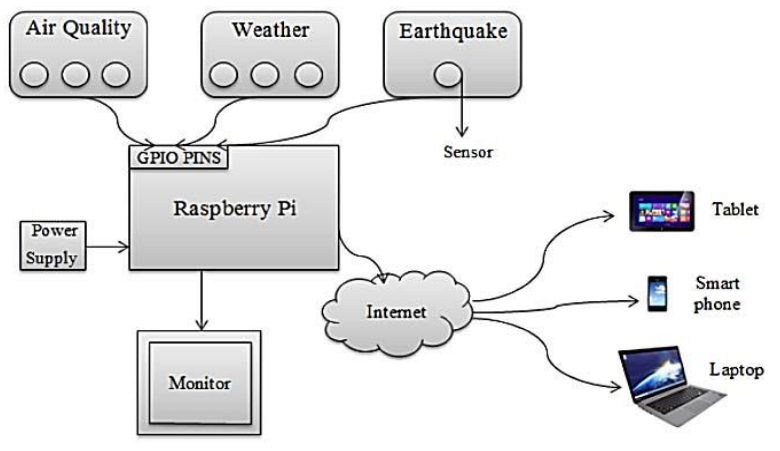

Fig 1: A schematic diagram of the system. [1]

The Fig 1 is proposed by the Ahmed Mohamed [1], as shown in the Fig1, we get the overview idea of the system. And used hardware used is Humidity sensors, temperature sensors, IR sensors and $\mathrm{CO}$ concentration sensor.

C. Balasubramaniyan and D. Manivannan.[2] developed the system Air Quality Monitoring System (AQMS) using Raspberry Pi and integrated with Internet of Things (IoT) gateway. In his work, generic sensor nodes/motes, sensor web nodes are designed using Raspbery $\mathrm{Pi}$ as processing unit, GrovePi+ electronic shields are used for interfacing sensors and $\mathrm{Wi}-\mathrm{Fi}$ is used as transceiver for data communication between web node and server. ThingSpeak is the IoT sever which is used in this paper.

Table 1. Raspberry Pi comparison with another SBC's [2].

\begin{tabular}{|c|c|c|c|c|c|}
\hline $\begin{array}{l}\text { Para } \\
\text { meter }\end{array}$ & $\begin{array}{l}\text { Raspberry } \\
\text { Pi }\end{array}$ & $\begin{array}{l}\text { Ardu } \\
\text { ino }\end{array}$ & $\begin{array}{l}\text { Intel } \\
\text { Galileo }\end{array}$ & $\begin{array}{l}\text { UDOO } \\
\text { NEO }\end{array}$ & $\begin{array}{l}\text { Beagle } \\
\text { bone }\end{array}$ \\
\hline $\begin{array}{l}\text { Proces } \\
\text { sor }\end{array}$ & $\begin{array}{l}\text { Broadcom } \\
\text { BCM2837 } \\
64 \mathrm{bit} \\
\text { ARMv7 } \\
\text { Quad Core } \\
1.3 \mathrm{Ghz}\end{array}$ & $\begin{array}{l}\text { ATM } \\
\text { EGA } \\
8, \\
\text { ATM } \\
\text { EGA } \\
1280\end{array}$ & $\begin{array}{l}\text { Intel } \\
\text { Quark } \\
\text { X1000 } \\
- \\
400 \mathrm{MH} \\
\text { z single } \\
\text { core }\end{array}$ & $\begin{array}{l}\text { Free scale } \\
\text { i.MX } \\
6 \text { SoloX } \\
1 \mathrm{GHz} \\
\text { ARM } \\
\text { Cortex- } \\
\text { A9 with } \\
\text { Cortex- } \\
\text { M4 }\end{array}$ & $\begin{array}{l}\text { AM335 } \\
91 \mathrm{GHz} \\
\text { ARM } \\
\text { Cortex- } \\
\text { A8 }\end{array}$ \\
\hline RAM & $1 \mathrm{~GB}$ & $\begin{array}{l}16- \\
32 \\
\mathrm{~KB}\end{array}$ & $\begin{array}{l}512 \mathrm{~Kb} \\
\text { on-chip } \\
\text { SRAM } \\
256 \mathrm{Mb} \\
\text { DRAM }\end{array}$ & $\begin{array}{l}512 \mathrm{MB} \\
\text { or } 1 \\
\text { GB(full) }\end{array}$ & $\begin{array}{l}512 \mathrm{MB} \\
\text { DDR3 } \\
\text { RAM }\end{array}$ \\
\hline $\begin{array}{l}\text { POW } \\
\text { ER }\end{array}$ & $10 \mathrm{~W}$ & $5 \mathrm{~W}$ & $15 \mathrm{~W}$ & $10 \mathrm{~W}$ & $15 \mathrm{~W}$ \\
\hline OS & $\begin{array}{l}\text { Raspbian, } \\
\text { Debian, } \\
\text { Fedora, } \\
\text { ARCH } \\
\text { Linux } \\
\text { ARM) and } \\
\text { FreeBSD }\end{array}$ & N. A & $\begin{array}{l}\text { Arduino } \\
\text { Linux } \\
\text { distribut } \\
\text { ion for } \\
\text { galileo. } \\
\text { Windriv } \\
\text { er } \\
\text { Rocket }\end{array}$ & $\begin{array}{l}\text { Android } \\
\text { Lollipop } \\
\text { and } \\
\text { Linux } \\
\text { UDOObu } \\
\text { ntu2 } \\
\text { (14.04 } \\
\text { LTS) }\end{array}$ & $\begin{array}{l}\text { Android } \\
\text { Debian, } \\
\text { Angstro } \\
\text { m, } \\
\text { yacto, } \\
\text { Fedora, } \\
\text { Ubuntu }\end{array}$ \\
\hline COST & $\$ 40$ & $\$ 30$ & $\$ 70$ & $\$ 65$ & $\$ 55$ \\
\hline
\end{tabular}

The author has done comparison of raspberry pi model with Single Board Computers (SBC's) as shown in Table 1. and conclusion of the above table is the latest raspberry pi i.e. raspberry pi model $3 \mathrm{~B}$ is cost-efficient SBC's from others and also compatible with complex task management.
In Survey paper [4] Priyanka S Lonare and Dr. Mahesh Kolte has implemented a secured wireless communication system of ARM embedded IoT server based on Raspberry Pi. In this paper the ESAYIoT sever is used as IoT server.

The Author's Hongyu Pei Breivold and Kristian Sandström [5] has discussed the clarification the specific quality attribute constraints within industrial automation, throw a light on various industrial IoT challenges and constraints, and discuss the potentials of utilizing some technical solutions to cope with these challenges. The challenges are Mixed criticality, Latency, Fault tolerance, Scalability with respect to data update cycles, Scalable collaboration, Functional safety, Industry-specific security challenge and Legacy long-lived industrial systems.

Charith Perera and Chi Harold Liu. [6] has done the survey on the Internet of Things with keeping in the mind of Industrial Marketplace. The paper explores a conceptual framework for context-aware product development and research in the IoT paradigm. The author has given an idea of exiting IoT product in market place. In this paper we also get an idea of significant research directions and trends.

In the Survey paper [7] $\mathrm{Li} \mathrm{Da} \mathrm{Xu} \mathrm{(Senior} \mathrm{Member,} \mathrm{IEEE)} \mathrm{and}$ $\mathrm{Wu} \mathrm{He}$, Shancang $\mathrm{Li}$ has explained the service oriented architecture of IoT and this architecture is divided into four layer as follows

1. Sensing layer, 2. Network layer, 3. Service layer and 4. Interface layer. This paper is also mention the IoT technologies and IoT application in the Industrial Automation. And some research challenges and future trends.

Song Han [8] has the discuss WirelessHART (Highly Addressable Remote Transducer) protocol which is also known as IEC-62591-2010 Standard in detail and he also explain the CoAP (Constrained Application Protocol) by designing the network infrastructure of wireless embedded Internet for industrial automation and author also presents the implementation of a prototype system which integrates WirelessHART mesh networks into the Internet and supports web-based monitoring and control services.

The role of Bluetooth Low Energy (BLE) is demonstrated by the author Gowrishankar.S [9]. in automating the Proximity based solutions for Internet of Things (IoT) by considering a real-world use case scenario. The author also shows how various disparate technologies are integrated together to form IoT paradigm. REST API protocol is used to developed the Android Application.

\section{MERIT AND DE-MERIT OF GIVEN SYSTEM}

\subsection{The Merits are discussed:}

The IIoT connects sensors to analytic and other systems to automatically improve performance, safety, reliability, and energy efficiency by:

1.Collecting data from sensors (things) much more cost e effectively than ever be-fore because sensors are often battery-powered and wireless

2.Interpreting this data strategically using big data analytic and other techniques to turn the data into actionable information.

3.Illustrating this significant information to the right person, it may operator or remote experts, and at the right time.

4.Delivering performance improvements when personnel take corrective action. 


\subsection{The challenges are as follow:}

1.Wi-Fi/Internet Connection is fluctuating which may create problems.

2.SMS/Email Alerts has to send but may have range problem. 3.Decision Making is very difficult as this is question of many life \& industry.

4.Wrong tool Selection for Development.

5.Sever Problems as controlling action becomes difficult.

6.Security and safety.
Managing Latency and Scalability of Data through Localization of Computation, Managing Mixed Criticality through System Partitioning, Managing Scalable and Secure Real-time Collaboration, Managing Fault Tolerance and Managing functional safety this all solution is discuss by author in [5].

\section{PROPOSED SYSYTEM}

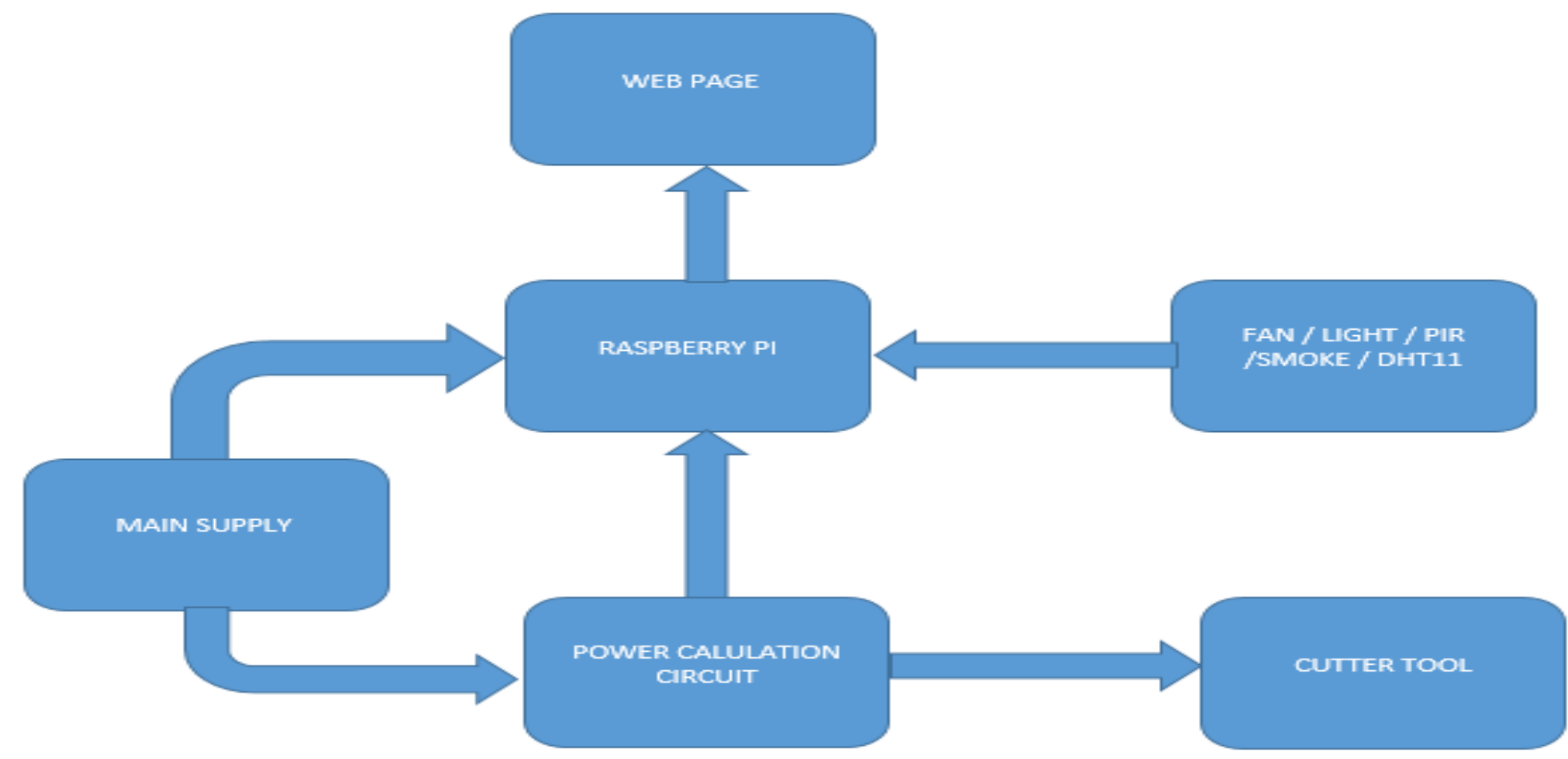

Fig 2: A Block diagram of the system

The block diagram of the system is shown in fig 2., here raspberry pi act as the computing system, the Light and Fan of the workshop is control through raspberry pi. The sensors are PIR and Smoke which is used to detect the movement of the people and Smoke sensor is used for the security purpose.

The main theme of the system to detect the age of blade by observing the current consumption. Accordingly, the alert is shown on web page.

\subsection{The hardware used in this system are as follows: \\ 1.Raspberry Pi \\ 2.Bulb \\ 3.MCP3208 (ADC) \\ 4.PCB Cutter Tool \\ 5.Hall sensor ACS712 \\ 6.PIR sensor \\ 7.Smoke sensor \\ 8.LM35}

The Environment of the workplace is monitored through web page. The energy is saved through by controlling the lights and Fans using PIR sensors, and by monitoring the power of Cutter tools and changing the blade of the cutter tool if it is damaged.

\subsection{Software used:}

1.Python

2.HTML/CSS/JQuery

\section{CSV format.}

4.FLASK

The overall programing is done with python, the sensors data is extracted using this software and stored. The HTML and CSS is used to design the web page of the system. The JQurey is small Java Script that is to fetch the data from Python. Flask is the framework that connect Python and HTML.

\section{DISCUSSION \& RESULT}

The signal conditioning circuit is developed to sense the AC current by the Hall Sensor and the observation is illustrated in table 2

Table 2. Observation of Various Load

\begin{tabular}{|c|c|}
\hline $\begin{array}{c}\text { AMPs(A) } \\
(\mathrm{y})\end{array}$ & $\begin{array}{c}\text { Voltage across signal } \\
\text { Conditioner }(\mathrm{V})(\mathrm{x})\end{array}$ \\
\hline 0.36 & 0.18 \\
\hline 0.74 & 0.31 \\
\hline 0.83 & 0.38 \\
\hline 1.12 & 0.45 \\
\hline 1.14 & 0.47 \\
\hline 1.26 & 0.59 \\
\hline 1.83 & 0.78 \\
\hline 2.27 & 1.01 \\
\hline 5.12 & 2.14 \\
\hline
\end{tabular}


By applying the various Load such as Bulbs, Machines etc. voltage across the signal conditioner is observe. The equation is form by the observation and as shown in equation 1

$$
y=2.3986 x-0.0517 \ldots \ldots \ldots \ldots \ldots \ldots \ldots . . . . . . .1
$$

where 0 ' $y$ ' is current and ' $x$ ' is voltage at signal conditioning output.

Observation is also made with Good and Bad Blade of Cutter tool, is as follows.

Table 3. Observation of Good Blade

\begin{tabular}{|c|c|}
\hline $\begin{array}{c}\text { AMPs(A) } \\
(\mathrm{y})\end{array}$ & $\begin{array}{c}\text { Voltage across signal } \\
\text { Conditioner }(\mathrm{V})(\mathrm{x})\end{array}$ \\
\hline 0.51 & 0.26 \\
\hline 0.65 & 0.28 \\
\hline
\end{tabular}

Table 4. Observation of Bad Blade

\begin{tabular}{|c|c|}
\hline $\begin{array}{c}\text { AMPs(A) } \\
(\mathrm{y})\end{array}$ & $\begin{array}{c}\text { Voltage across signal } \\
\text { Conditioner }(\mathrm{V})(\mathrm{x})\end{array}$ \\
\hline 0.51 & 0.26 \\
\hline 0.75 & 0.35 \\
\hline
\end{tabular}

The first observation in both the table i.e. table 2 and table 3 is the current consume by the motor with no load that is with cutting material. When blade is sharp and in good condition the current consume by motor is $0.65 \mathrm{Amps}$. When blade blond and bad condition the current consume is $0.75 \mathrm{Amps}$.

The hardware implementation is shown in fig1. Where LM35, hall sensors, motion sensor and smoke sensor are mounted on $\mathrm{PCB}$, which sends the data to the raspberry pi through the connector.

Here data is collected from various sensors, through python language used in raspberry pi. The data can be monitored through the web page as shown in the fig4.

Temperature and the Current consumption of cutter tool is monitored as shown in fig4 and as option to download the data. Also, the data can be viewed in tabular format as illustrated in fig5. And in graphical format too as shown in fig6.

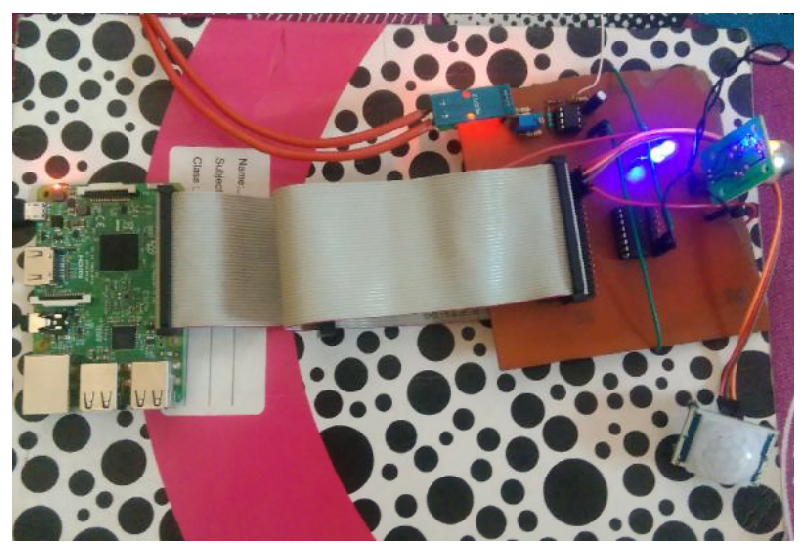

Fig 3: Hardware of the system

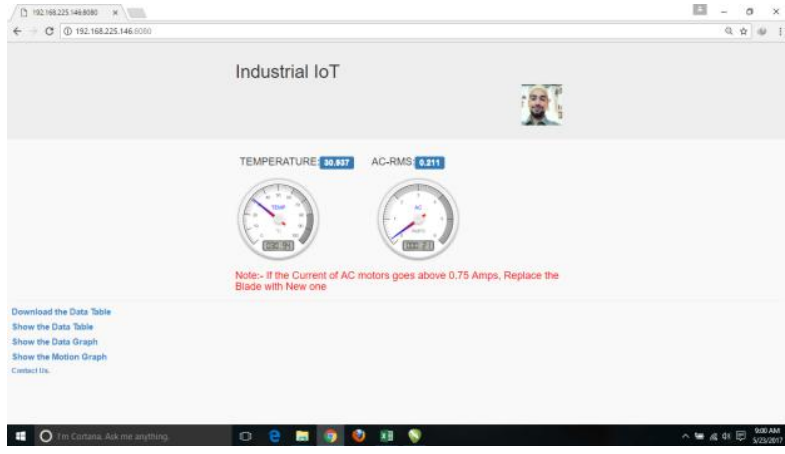

Fig 4: Webpage of the system

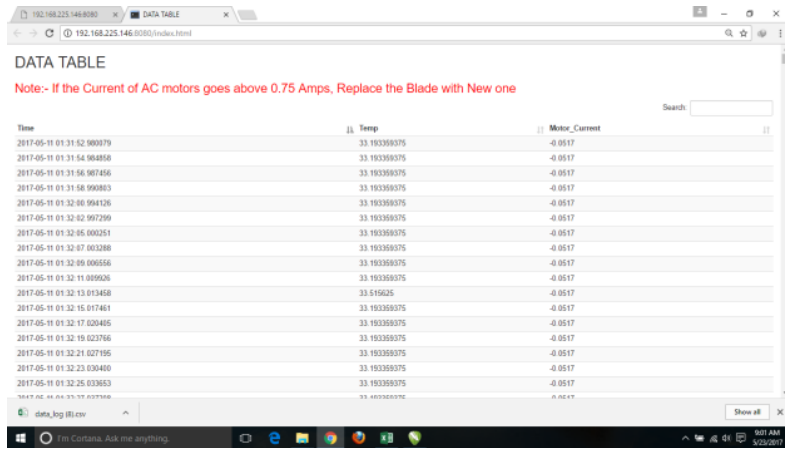

Fig 5: Data Shown in Tabular Format

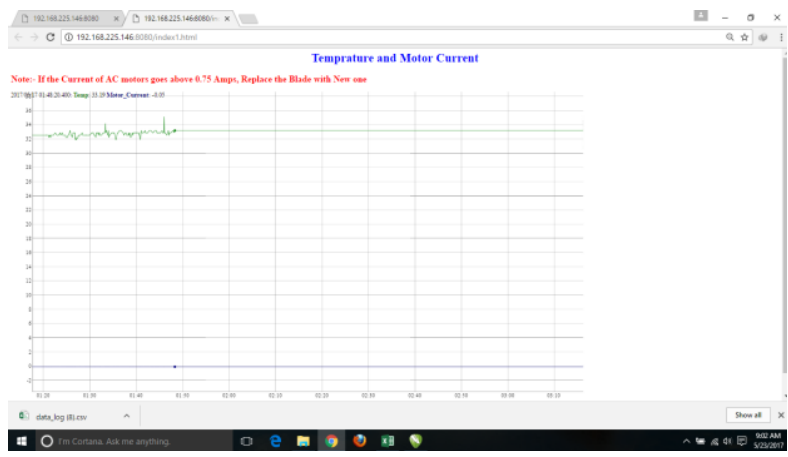

Fig 6: Data Shown in Graphical Format

\section{CONCLUSION}

As mentioned in the Section II, we get the information of the SOA-IoT that is Service Oriented Architecture IoT. We also get the idea about the System overview of raspberry pi. We are aware from the new trends in the Industrial automation and IoT technologies. The authors also discuss the various challenges in the IoT and also in Industrial automation constrain. How handle this challenge is also discussed by the authors. The authors also survey the industrial marketplace considering the IoT perspective. The embedded internet network was discussed by the author in which IEC 62591 that is wirelessHART standard was mention in detail. A good comparison done by the author in which why to use raspberry pi in industrial propose is explained. As raspberry pi is small in size and also consumed less power along with doing complex processing of collected data.

There are lots of wastage in energy at workstation and industries by hiding the real status of the installed system through workers or may be the middle hierarchy of industries.

This system try to minimize the energy waste by providing sufficient information to the owner or top level hierarchy persons via remotely and can be made appropriate decision. It 
also helps to analyze the overview consumption of power and material requirement. In this system, blade aging of the cutter tool is monitored by observing the energy consumption of cutter tools.

\section{ACKNOWLEDGMENTS}

The author wish to express his special thanks to Prof. D. D. Ahire for his consistence support for this project. The author also thanks to the Matoshri College of Engineering and Research center, Nashik, India for providing the infrastructure to carry out this work.

\section{REFERENCES}

[1] Mohammad Ibrahim, Abdelghafor Elgamri and Ahmed Mohamed, Internet of Things based Smart Environmental Monitoring using the Raspberry-Pi Computer,ISBN: 978-1-4673-6832-22015 IEEE.Ding, W. and Marchionini, G. 1997 A Study on Video Browsing Strategies. Technical Report. University of Maryland at College Park.

[2] Mauro C. Balasubramaniyan* and D. Manivannan, IoT Enabled Air Quality Monitoring System (AQMS) using Raspberry Pi,Indian Journal of Science and Technology, Vol 9(39), DOI:10.17485/ijst/2016/v9i39/90414, October 2016.

[3] Priyanka S Lonare1, Dr. Mahesh Kolte2, A Raspberry Pi Based Global Industrial Process Monitoring through Wireless Communication, International Journal of Advanced Research in Computer and Communication Engineering,Vol. 5, Issue 9, September 2016.
[4] Hongyu Pei Breivold,Kristian Sandstrm, Internet of Things for Industrial Automation Challenges and Technical Solutions, 2015 IEEE International Conference on Data Science and Data Intensive Systems,978-1-5090-0214-6/15 31.002015 IEEE,DOI 10.1109/DSDIS.2015.11.

[5] S. CHARITH PERERA, CHI HAROLD LIU and MIN CHEN, A Survey on Internet of Things From Industrial Market Perspective,2169-3536 2015 IEEE.

[6] Li Da Xu (Senior Member, IEEE), Wu He, Shancang Li, Internet of Things in Industries: A Survey, Citation information: DOI 10.1109/TII.2014.2300753, IEEE Transactions on Industrial Informatics.

[7] Song Han \& Yi-Hung Wei,Deji Chen, Mark Nixon, Eric Rotvold, Aloysius K. Mok, Building Wireless Embedded Internet for Industrial Automation.

[8] Gowrishankar.S, Madhu.N and T.G.Basavaraju, Role of BLE in Proximity Based Automation of IoT:A Practical Approach,2015 IEEE Recent Advances in Intelligent Computational Systems (RAICS) | 10-12 December 2015 | Trivandrum.

[9] Ashwini Deshpande, Sangita Sanap, Industrial Automation using Internet of Things (IOT), International Journal of Advanced Research in Computer Engineering Technology (IJARCET) Volume 5 Issue 2, February 2016.

[10] HTML5 Tutorial https://www.w3schools.com/html/ 